\title{
Effects of Age and Loading Velocity on the Delamination Strength of the Human Aorta
}

\author{
L. Horný*, L. Roubalová ${ }^{\S}$, Z. Petřivý ${ }^{\S}$, H. Chlup ${ }^{\S}$, J. Kronek ${ }^{\S}$ P. Tichý ${ }^{\S}$, T. Adámek ${ }^{\#}$, \\ A. Blanková $a^{\#}$, and T. Such $\dot{y}^{\dagger}$ \\ ${ }^{*}$ Faculty of Mechanical Engineering \\ Czech Technical Universtiy in Prague \\ Technická 4, 16000 Prague, Czech Republic \\ email: lukas.horny@fs.cvut.cz \\ \# Department of Forensic Medicine and Toxicology \\ Regional Hospital Liberec \\ Husova 357/10, 46063 Liberec, Czech Republic \\ $\dagger$ Institute of Rock Structure and Mechanics of the Czech Academy of Sciences \\ V Holešovičkách 94/41, 18209 Prague, Czech Republic
}

\begin{abstract}
Delamination strength is the mechanical property which plays a key role in the pathological process referred to as Arterial Dissection. This dissection, known especially for its occurrence in the thoracic aorta, is manifested by a separation of the layers of an artery wall, and may end with total rupture and internal haemorrhaging. Although its incidence is relatively rare, from 3 to 6 cases per 100000 per year, it is a life-threating disease with a significant lethality [1-3].

The exact conditions under which the dissection is initiated, and as a crack propagates through the arterial wall, remain an open topic in computational as well as experimental mechanics. The aim of our study is to contribute to the deepening of our knowledge of Arterial Dissection, by collecting experimental data which is suitable for the purpose of showing how the delamination strength measured in the peeling experiments depends on age and anatomical location. In addition to the effects of age and location, our study also focuses on the effect of loading rate.
\end{abstract}

The experimental branch of our research is complemented by a computational modelling of the delamination interface, in which we are looking for a numerical characterization of the material parameters describing discontinuity propagation. An XFEM model of the peeling experiment is built in Abaqus, which in our approach plays the role of the regression analysis, incorporating the cohesive zone (CZ) in order to model the delaminating arterial layers. The main objective is to obtain a detailed description of a set of constitutive parameters, which would be age- and location-specific.

Our present data suggest that delamination strength strongly depends on age, and furthermore, the anatomical site also seems to be a significant factor. On the other hand, the loading velocity does not cause significant changes in results.

This study has been supported by the Czech Science Foundation in the project GA20-11186 entitled "Mechanics of Arterial Delamination and Crack Propagation".

\section{REFERENCES}

[1] C.A. Nienaber, and R.E. Clough, "Management of acute aortic dissection", The Lancet, $\mathbf{3 8 5}, 800$ $811(2015)$.

[2] C. Olsson, S. Thelin, E. Ståhle, A. Ekbom, and F. Granath, "Thoracic aortic aneurysm and dissection: Increasing prevalence and improved outcomes reported in a nationwide populationbased study of more than 14000 cases from 1987 to 2002", Circulation, 114, 2611-2618 (2006).

[3] I. Mészáros, J. Mórocz, J. Szlávi, J. Schmidt, L. Tornóci, L. Nagy, and L. Szép, „Epidemiology and clinicopathology of aortic dissection: A population- based longitudinal study over 27 years“, Chest, 117 1271-1278 (2000). 